Draft VERSion MAY 27, 2018

Preprint typeset using $\mathrm{LAT}_{\mathrm{E}} \mathrm{X}$ style emulateapj v. 08/22/09

\title{
THE DISCOVERY OF A VERY FAINT X-RAY TRANSIENT IN THE GLOBULAR CLUSTER M15
}

\author{
Craig O. Heinke ${ }^{1,2}$, Haldan N. Cohn $^{3}$, Phyllis M. Lugger $^{3}$ \\ Draft version May 27, 2018
}

\begin{abstract}
We have identified an X-ray transient (hereafter M15 X-3) in the globular cluster M15 from an archival Chandra grating observation. M15 X-3 appears at an X-ray luminosity of $6 \times 10^{33} \mathrm{ergs} \mathrm{s}^{-1}$ with a spectrum consistent with an absorbed power law of photon index $1.51 \pm 0.14$. The object is identifiable in archival Chandra HRC-I observations with an X-ray luminosity of $2-6 \times 10^{31} \mathrm{ergs} \mathrm{s}^{-1}$ and apparently soft colors, suggesting a neutron star low-mass X-ray binary in quiescence. We also observe it in outburst in a 2007 Chandra HRC-I observation, and in archival 1994-1995 ROSAT HRI observations.

We identify a likely optical/UV counterpart with a (possibly transient) UV excess from archival HST data, which suggests a main sequence companion. We argue that M15 X-3's behavior is similar to that of the very faint X-ray transients which have been observed in the Galactic Center. We discuss several explanations for its very low X-ray luminosity, with the assumption that we have detected its companion. M15 X-3's uniquely low extinction and well-determined distance make it an excellent target for future studies.
\end{abstract}

Subject headings: binaries : X-rays — stars: neutron — globular clusters: individual (NGC 7078) accretion disks

\section{INTRODUCTION}

Low-luminosity X-ray sources in globular clusters were identified with Einstein (Hertz \& Grindlay 1983) as a separate class of X-ray sources from bright $\left(10^{36}<L_{X}<10^{38} \mathrm{ergs} / \mathrm{s}\right)$ LMXBs containing neutron stars (NSs). HG83 suggested that these systems were primarily cataclysmic variables $(\mathrm{CVs})$, with similar accretion rates to the LMXBs but potential energy wells 1000 times shallower, but also including some NS LMXBs in quiescence (qLMXBs). However, Verbunt et al. (1984) argued that the brightest low- $L_{X}$ systems $\left(L_{X}>10^{33}\right.$ ergs/s) were too bright to be CVs, and must be qLMXBs.

Many low- $L_{X}$ cluster sources have now been observed with the Chandra $X$-ray Observatory, and the answer has been mixed; some of the brighter low- $L_{X}$ systems have been identified with qLMXBs, some with CVs (e.g. in 47 Tuc, Grindlay et al. 2001), while others remain unidentified. The brightest few low- $L_{X}$ systems $\left(5 \times 10^{33}<L_{X}<10^{35} \mathrm{ergs} / \mathrm{s}\right.$, generally transient $)$ remain a puzzle for either explanation. These objects are more luminous than known CVs, and are brighter than qLMXBs emitting X-rays from heat stored in the crust between accretion events. However, they are fainter than expected for X-ray binaries undergoing outbursts driven by the standard disk instability model (King 2000). Eight such systems may have been seen, with various X-ray telescopes (Einstein, ROSAT, Chandra), in several globular clusters, but none have been studied in great detail (Heinke et al. 2008, in prep). Only one of them (Source B in NGC 6652, Heinke et al. 2001) shows strong evidence

\footnotetext{
${ }^{1}$ University of Alberta, Dept. of Physics, Room \#238 CEB, 11322 - 89 Avenue, Edmonton, AB, T6G 2G7, Canada; cheinke@phys.ualberta.ca

${ }^{2}$ University of Virginia, Dept. of Astronomy, PO Box 400325, Charlottesville, VA 22903

${ }^{3}$ Astronomy Dept., Indiana University, 727 East 3rd St., Bloomington, IN 47405
}

for being a quiescent low-mass X-ray binary, though its accretion history is uncertain.

Thirty very faint X-ray transients (VFXTs) have been identified, mostly in the Galactic Center region, having peak X-ray luminosities $10^{34}<L_{X}<10^{36}$ ergs/s and quiescent $L_{X}$ at least a factor of 10 lower (Muno et al. 2005b; Sakano et al. 2005; Wijnands et al. 2006; Degenaar \& Wijnands 2008). Explanation of their very low outburst luminosities and low inferred timeaveraged mass transfer rates $\left(\dot{\mathrm{M}} \lesssim 10^{-12} M_{\odot}\right)$ may require new paths of binary evolution. These objects are now being well-studied with Chandra, XMM-Newton, and Swift through monitoring of the Galactic Center, where the majority have been seen. There is some overlap between these VFXTs and the class of low-luminosity X-ray bursters (Cocchi et al. 2001; Cornelisse et al. 2002; Hands et al. 2004), which indicates that at least some of these systems are low-mass X-ray binaries. Some low-luminosity X-ray bursters show extremely short Xray outbursts (Wiinands et al. 2007), while at least one VFXT, XMMU J174716.1-281048, shows years-long activity at $L_{X}=$ few $\times 10^{34} \mathrm{ergs} / \mathrm{s}$ (Del Santo et al. 2007). We are undertaking a program to search for and study VFXTs in globular clusters, which provide opportunities to study the optical, ultraviolet, and soft X-ray emission not available in observations of the Galactic Center.

The globular cluster M15 (NGC 7078) is of particular interest for X-ray and optical astronomy. M15 is a massive cluster that shows signs of advanced core collapse, with a radial stellar density profile showing a steep cusp to within the central arcsecond (Lauer et al. 1991; Guhathakurta et al. 1996; Sosin \& King 1997). M15 shows a central concentration of relatively underluminous mass, that may be composed of compact objects (such as heavy white dwarfs and neutron stars, Dull et al. 1997, 2003; Baumgardt et al. 2003) or an intermediate-mass black hole (Gerssen et al. 2002). 

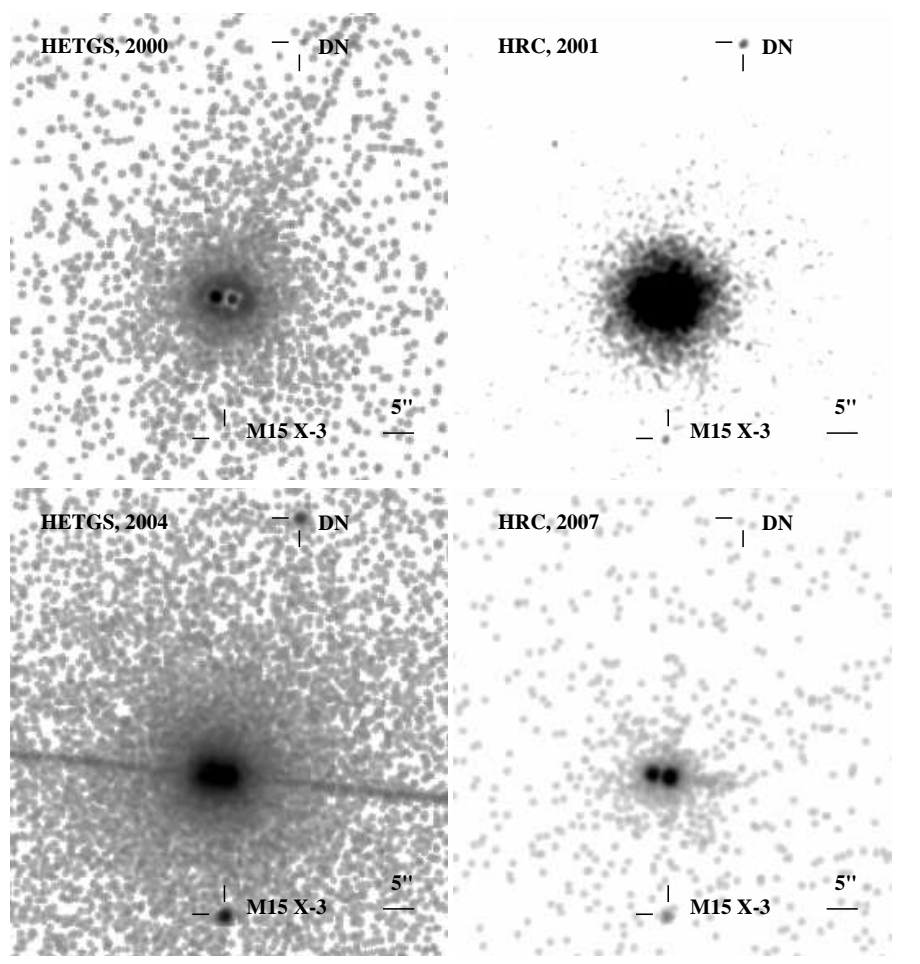

Fig. 1.- Chandra images of M15, from 2000 ACIS-HETGS, 2001 HRC-I, 2004 ACIS-HETGS, and 2007 HRC-I data. All data are smoothed with a gaussian of kernel $0.75^{\prime \prime}$. The dwarf nova (DN) identified by Hannikainen et al. (2005) and M15 X-3 are marked. Readout streaks can be seen at position angles $\sim 30$ and $\sim 100$ degrees from the saturated LMXBs in the HETGS data. The greyscale is chosen to emphasize M15 X-3 in each image.

Its high central density also makes it a likely location for stellar encounters, which should produce LMXBs, CVs, and millisecond radio pulsars. Studies in the X-ray (requiring the use of Chandra), optical, and radio have indeed identified two bright LMXBs (White \& Angelini 2001), several CVs (Charles et al. 2002; Hannikainen et al. 2005; Dieball et al. 2007) and eight radio pulsars (Anderson 1993). We use a distance of $10.3 \mathrm{kpc}$ (van den Bosch et al. 2006) and a column density of $N_{H}=4.6 \times 10^{20} \mathrm{~cm}^{-2}$ (Janulis 1992) in this paper. Here we report the detection of a very faint X-ray transient in the globular cluster M15.

\section{CHANDRA DATA ANALYSIS}

We reduced all 6 Chandra observations of M15 in the Chandra data archive ${ }^{4}$ (see Table 11). All data were reduced and analyzed using CIAO 3.4.1. For the ACIS exposures, we generated new bad pixel files, reprocessed the ACIS data removing pixel randomization, and filtered on grade and status. For all data, we searched for periods of high, flaring background, but found none. ${ }^{5}$

Several X-ray sources are apparent in the images (Fig. 11), including the two bright LMXBs reported by White \& Angelini (2001), two faint X-ray sources (a dwarf nova, DN, and planetary nebula, PN) reported by Hannikainen et al. (2005), and the transient reported here. After trying various detection strategies, we chose

\footnotetext{
${ }^{4}$ http://cda.harvard.edu/chaser/mainEntry.do

${ }^{5}$ A smooth $60 \%$ rise in the background over the length of Obs_ID 2413 is still small compared to the scattered X-ray background from the LMXBs.
}

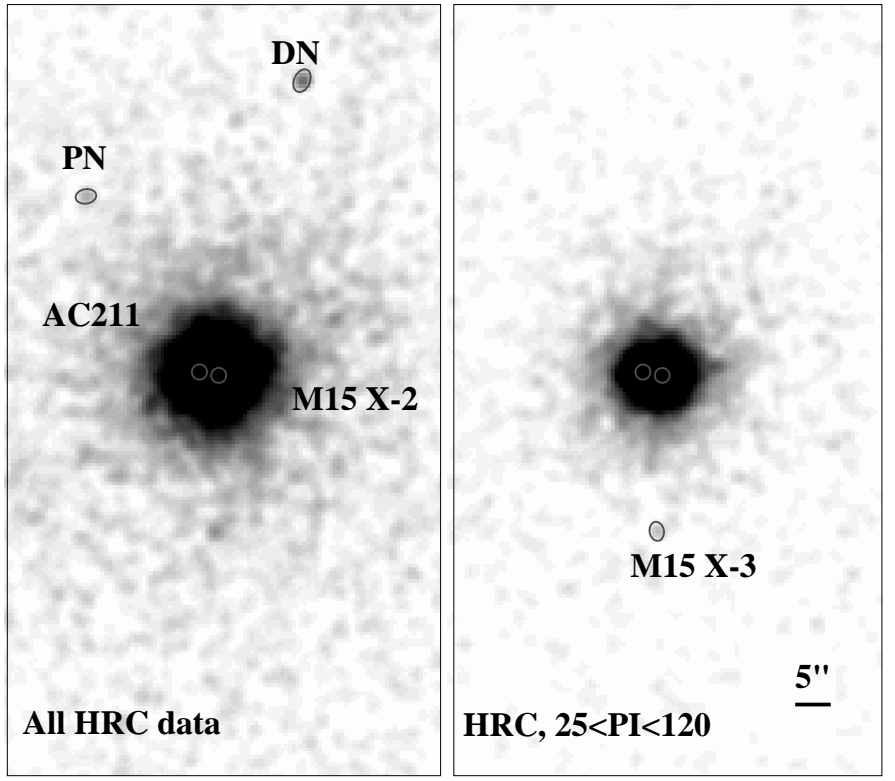

Fig. 2.- Combined 2001 Chandra HRC images of M15, including all good events (left) and only a soft subset $(25<\mathrm{PI}<120$, right), binned by 2 and smoothed with a gaussian of kernel $0.75^{\prime \prime}$. Ellipses encircle sources detected by WAVDETECT in each image, which are labeled $(\mathrm{PN}=$ planetary nebula, $\mathrm{DN}=$ dwarf nova $)$.

the following strategies, all on $2^{\prime} \times 2$ ' images centered on M15's core. To locate the bright LMXBs in the HETGS observations, we ran the CIAO detection program WAVDETECT without an energy band restriction. We did this in order to ensure the bright LMXBs retained as many of their photons as possible, as the zerothorder images of the two bright LXMBs show substantial pileup. ${ }^{6}$ To detect the other faint X-ray sources in M15 during the ACIS observations, we ran WAVDETECT in the $0.5-7 \mathrm{keV}$ and $0.5-2 \mathrm{keV}$ ranges.

We reprojected the three 2001 HRC observations and merged them to increase sensitivity. We ran WAVDETECT on two images; the full HRC dataset, and a dataset filtered to include only softer photons by including only events in pulse-invariant (PI) channels between 25 and 120 (cf. Cameron et al. 2007). We binned the HRC data by a factor of two. We show both HRC images of M15 in Fig. 2.

We chose WAVDETECT detection thresholds of 1e-6, a conservative limit which should produce much less than 1 false source in each image. We identified no sources in the 2000 HETGS data besides the two bright LMXBs. In the combined, full-energy HRC image from 2001 we identified two sources noted by Hannikainen et al. (2005), identified as a dwarf nova (DN, their source C) and a planetary nebula (source D; PN K648), and determined accurate positions for the two LMXBs. In the 2004 data we identify a new transient (hereafter M15 X-3), as well as the DN. In the 2007 HRC-I data we again detect the new transient, along with the two bright LMXBs (as reported by Heinke et al. 2007).

Our WAVDETECT run on the filtered HRC data did not detect the PN or DN, but did detect a faint (8-count) source consistent with the position of M15 X-3 (Fig. 2).

\footnotetext{
${ }^{6}$ Pileup is the recording of more than one photon as part of the same event during a single frametime, which leads to distortion of the energy spectrum and loss of events (Davis 2001).
} 
No other, spurious, sources were detected in these detection runs, so we believe that we have indeed detected M15 X-3 in quiescence. Its detection in the filtered HRC data suggests that its quiescent spectrum is very soft, as is typically observed for NS LMXBs in quiescence.

We aligned our astrometry with the best radiodetermined position of AC 211 (Kulkarni et al. 1990) for the HRC frames, precessing from B1950 to J2000 using the NED precession calculator. ${ }^{7}$ We derive our most accurate position for M15 X-3 using the offset from AC 211 in the 2007 HRC-I data, in Table 3 . Positions derived from the marginal HRC-I 2001 detection and 2004 HETGS detection are consistent within $3 \sigma$ with this detection, but are subject to systematic errors (due to the marginal 2001 detection and effects of pileup on the derived position of AC 211 in 2004). Our listed positions for the other sources are derived from the offsets from AC 211 using the 2001 HRC-I data.

We do not detect sources A and B from Hannikainen et al. (2005), though we do not utilize their psf-subtraction method. We note that the HRC $\mathrm{X}$-ray source identified with a $\mathrm{PN}$ by Hannikainen et al. $(2005)$ is undetected in the 2004 HETGS dataset, suggesting that it has a soft spectrum (though not as soft as M15 X-3 in quiescence) or is variable. We defer further analysis of the other X-ray sources in M15 to future work.

\subsection{X-ray Spectral Analysis}

Transiently accreting NSs in quiescence are usually seen to have soft, blackbody-like X-ray spectra, often accompanied by a harder X-ray component generally fit by a power-law of photon index 1-2 (Campana et al. 1998). The harder component is of unknown origin; an effect of continued accretion, or a shock from a pulsar wind have been suggested (Campana et al. 1998). The blackbodylike component is generally understood as the radiation of heat from the NS surface. This heat is produced by deep crustal heating during accretion, and is radiated by the crust on a timescale of $10^{4}$ years, producing a steady quiescent thermal NS luminosity (Brown et al. 1998; Campana et al. 1998; Haensel \& Zdunik 1990). At higher luminosities (above $\sim 2 \times 10^{33} \mathrm{ergs} / \mathrm{s}$ ), the spectra of faint accreting NSs are generally dominated by the hard component (e.g. Jonker et al. 2004). The spectra of cataclysmic variables can often be represented by one or more hot plasma components, e.g. the MEKAL models in XSPEC (Liedahl et al. 1995), or, with low statistics, a power-law of photon index 1-2.

ACIS: We extract a spectrum, background and response files for M15 X-3 from the 2004 ACIS data, using the CIAO specextract script. The spectrum can be well fit by an absorbed power-law of photon index $\Gamma=1.51 \pm 0.14$ at the cluster's $N_{H}$ value (see Table 4 Figure 3). Adding a hydrogen-atmosphere neutron star atmosphere model (the NSATMOS model, Heinke et al. 2006) does not improve the fit, but we can place constraints on the luminosity in a NSATMOS component of $L_{X}(0.5-10 \mathrm{keV})<8.9 \times 10^{32} \mathrm{ergs} / \mathrm{s}$. A hot thermal plasma model (MEKAL) can also describe the spectrum adequately, with a temperature of $14_{-6}^{+17} \mathrm{keV}$.

\footnotetext{
7 http://nedwww.ipac.caltech.edu/forms/calculator.html
}

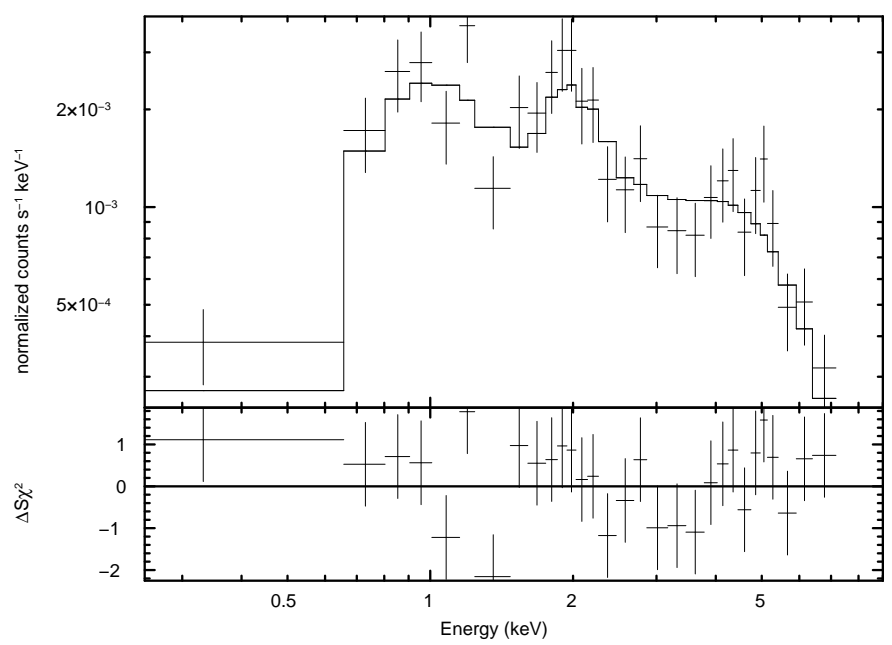

Fig. 3.- Top: Chandra 2004 HETGS X-ray spectrum (data and best-fit absorbed power-law model) of M15 X-3. Bottom: Residuals to best fit.

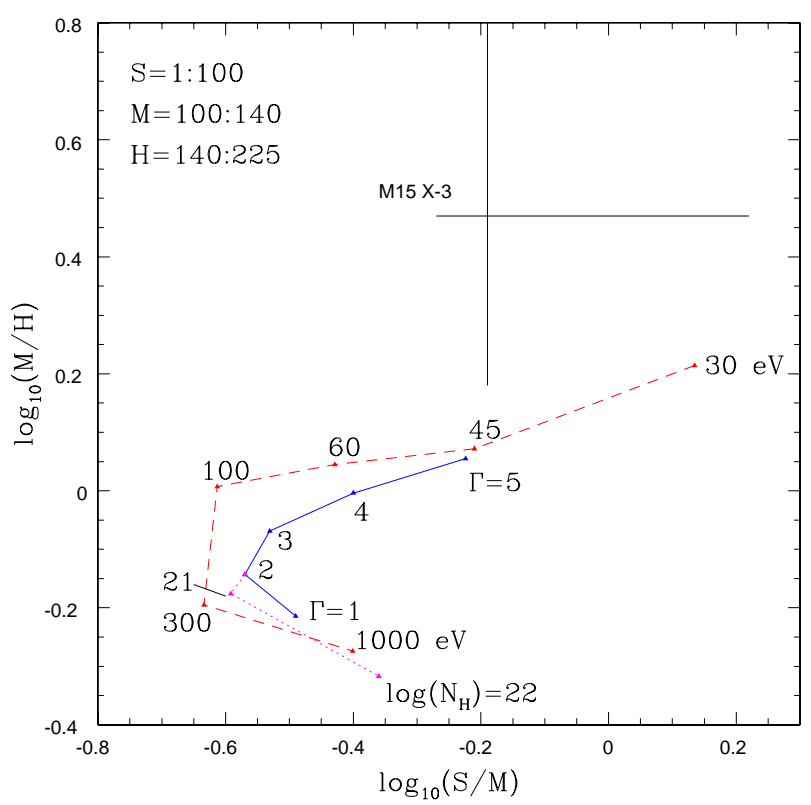

FIG. 4.- HRC-I hardness ratios for various power-law spectra (photon index $\Gamma=1$ to 5 , blue solid line) and blackbody spectra ( $\mathrm{kT}=30$ to $1000 \mathrm{eV}$, red dashed line) using the HRC-I response matrix. We show the effect of increasing the $N_{H}$ columns from $4.6 \times 10^{20}$ to $10^{21}$ or $10^{22} \mathrm{~cm}^{-2}$ for the power-law with photon index 2 (magenta dotted line). M15 X-3's quiescent colors and 1- $\sigma$ uncertainties are also plotted, suggesting a very soft spectrum.

$H R C$ : HRC data is not amenable to standard spectral fitting, but hardness ratios can be computed and some spectral information extracted from comparison with the response matrix provided by the Chandra X-ray Center. ${ }^{8}$ Only 13 counts are present within a $1^{\prime \prime}$ source region in the combined 2001 HRC data, of which $\sim 3$ are probably background. Given these limitations, the ratios of counts in PI channels 1:100, 100:140, and 140:255 suggest a power-law photon index $>4$ (Fig. 4). This suggests

\footnotetext{
8 See http://cxc.harvard.edu/cal/Hrc/RMF/
} 


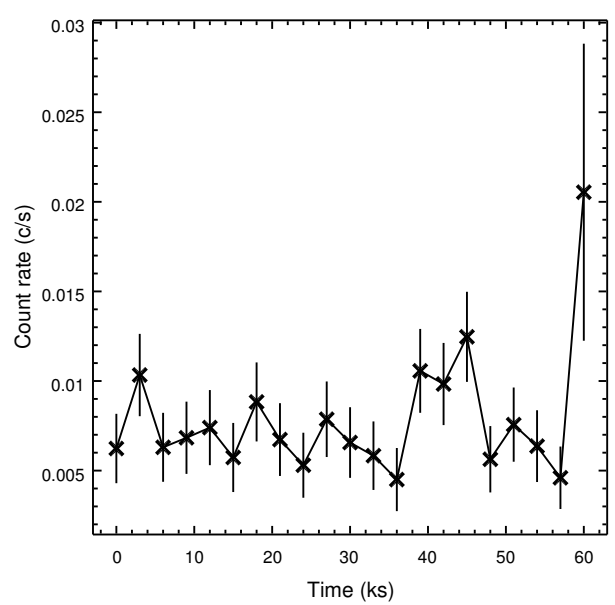

FIG. 5. - Background-subtracted lightcurve of M15 X-3 during the 2004 ACIS-S/HETGS observation.

a very soft source, such as is often seen from quiescent LMXBs Jonker et al. 2004), although we caution that the statistics are very poor and the response matrix uncertain.

We estimate the $0.5-10 \mathrm{keV}$ X-ray luminosity of M15 $\mathrm{X}-3$ in quiescence as $6 \times 10^{31} \mathrm{ergs} / \mathrm{s}$ (for a $\Gamma=2$ powerlaw) to $2 \times 10^{31} \mathrm{ergs} / \mathrm{s}$ (for a blackbody of $k T=110 \mathrm{eV}$ ). A hydrogen atmosphere model (NSATMOS, Heinke et al. 2006) with $\mathrm{T}=57 \mathrm{eV}$ and $10 \mathrm{~km}$ radius provides a soft spectrum and comparable observed countrate, and a bolometric NS luminosity of $8 \times 10^{31} \mathrm{ergs} / \mathrm{s}$. Such soft $\mathrm{X}$-ray sources would be hard to detect in zeroth-order HETGS images; in the 2000 HETGS observation, only 2 or 1 counts are expected, given the powerlaw or blackbody spectral models above. Therefore the nondetection of M15 X-3 in the 2000 data is not surprising.

\subsection{X-ray Variability}

To assess variability within the 2004 observation, we produced barycentered lightcurves of M15 X-3 and analyzed them using HEASARC's XRONOS software ${ }^{9}$. We show a lightcurve (binned at 3000 seconds) of M15 X-3 in Fig [5. A $\chi^{2}$ test on this lightcurve gives a probability of 0.02 that M15 X-3 is constant during the 2004 observation. The power spectrum shows red noise below $3 \times 10^{-3} \mathrm{~Hz}$, but no obvious periodic signals.

\section{ARCHIVAL X-RAY DATA ANALYSIS}

Several previous X-ray missions have had sufficient resolution to separate M15 X-3 from the other 2 LMXBs in M15 (though not to separate the two bright LMXBs). We analyze archival data from ROSAT HRI \& PSPC, ASCA SIS, EXOSAT HRI, and Einstein HRI observations to look for evidence of M15 X-3. First we consider the constraints possible over the past 12 years from RXTE.

\subsection{RXTE All-Sky Monitor Analysis}

We used data from the RXTE All-Sky Monitor ${ }^{10}$ to constrain M15 X-3's flux history. The known M15 LMXBs (AC 211 and M15 X-2) together contribute

\footnotetext{
${ }^{9}$ http://heasarc.gsfc.nasa.gov/docs/xanadu/xronos/xronos.html

10 http://xte.mit.edu
}

roughly $4 \times 10^{36} \mathrm{ergs} / \mathrm{s}(2-10 \mathrm{keV})$, and their combined luminosity is seen to be fairly stable to within a factor of two, apart from short periods when M15 is near the Sun and the data quality is poorer (White \& Angelini 2001). Thus, an outburst from M15 X-3 would have to be of similar luminosity to be potentially discernible.

M15 X-3's observed $L_{X}$ in 2004 might be the peak of an outburst, or the tail of an outburst with a higher peak $L_{X}$. M15 is not conclusively detected by the ASM during April 2004, at a time when M15 X-3 was active. A 2-sigma limit to the flux of M15 during the two weeks preceding this observation is $3 \times 10^{36} \mathrm{ergs} / \mathrm{s}$ in the $2-10$ keV band, assuming a Crab-like spectrum; this is consistent with the X-ray luminosity from M15 over the RXTE era. A rough limit on the flux earlier in 2004, and over the RXTE era generally, is $6 \times 10^{36} \mathrm{ergs} / \mathrm{s}$. This suggests that any outbursts from M15 X-3 in the RXTE era did not significantly exceed $3 \times 10^{36} \mathrm{ergs} / \mathrm{s}$, at least for more than a few weeks (but see below).

Our 2007 HRC-I observation was taken 2 months after the highest fluxes ever recorded from M15 by the RXTE ASM $\left(50 \mathrm{mCrab},{ }^{11}\right.$ or $\left.1.5 \times 10^{37} \mathrm{ergs} / \mathrm{s}\right)$. It is possible that these high fluxes were produced by another X-ray source besides the two previously known LMXBs, AC 211 and M15 X-2. Our HRC-I observation finds M15 X3 active, but not brighter than in the three other active episodes. Thus we do not clearly resolve whether M15 $\mathrm{X}-3$ or another source may have produced the high flux episode from M15.

\subsection{ROSAT Data Analysis}

Two lengthy ROSAT HRI observations of M15 exist in the HEASARC archive (PI: Grindlay), in addition to two shorter PSPC observations (we use only the on-axis one); see Table 1. The ROSAT XRT/HRI combination has a FWHM of 3", sufficient to resolve M15 X-3 from the two bright X-ray binaries in the core of M15, and an absolute positional accuracy of 5.3" (Deutsch et al. 1998). Although our detection algorithms do not identify a source at this location, a weak source is visible to the reader in the two HRI images (see Fig. 6, left), with $165 \pm 21$ and $35 \pm 9$ counts above the local background within 5" circles. Assuming an absorbed powerlaw spectrum of photon index 1.5 such as seen in 2004, we estimate $L_{X}(1994,0.5-10 \mathrm{keV}) \sim 7.4 \times 10^{33} \mathrm{ergs} / \mathrm{s}$ and $L_{X}(1995,0.5-10 \mathrm{keV}) \sim 7.7 \times 10^{33} \mathrm{ergs} / \mathrm{s}$.

\subsection{Other HEASARC Data Analysis}

For the remaining high-resolution imaging X-ray datasets (ASCA SIS, ROSAT PSPC, EXOSAT CMA, and Einstein HRI), we can provide only upper limits to M15 X-3's flux. The first step is to clarify whether the known LMXBs are those producing the detected source in prior observations. Figure 7 shows images from ASCA, EXOSAT, and Einstein images. In each case the blind pointing accuracy of the telescope is thought to be much better than $22^{\prime \prime}$ at $90 \%$ confidence, and in each case (see below) the detected source is consistent with the positions of the known bright LMXBs, and not with M15 $\mathrm{X}-3$.

The background is in each case dominated by the point spread functions of the nearby bright LMXBs. For sim-

\footnotetext{
11 Noted on http://xte.mit.edu/xte_anno.html
} 


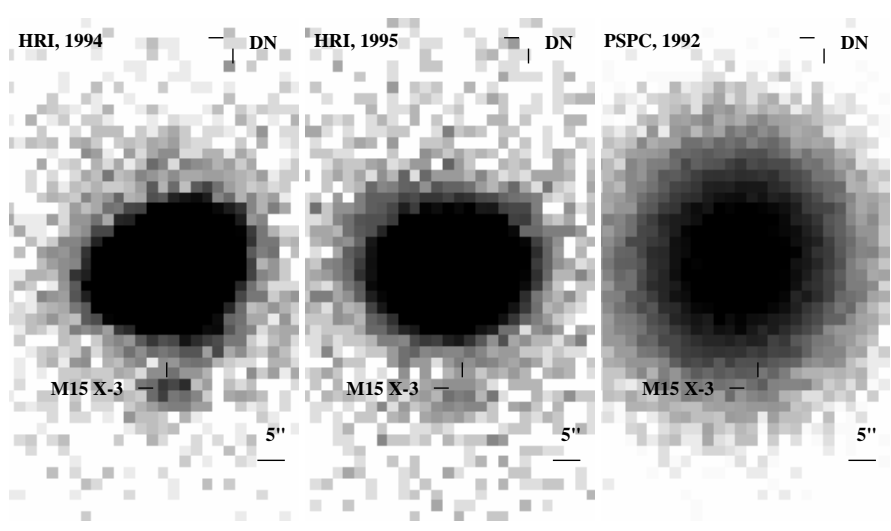

Fig. 6. - ROSAT images of M15. A weak source can be seen at a position consistent with M15 X-3, 22.6" south of the two bright LMXBs, in the two HRI images. The image scaling is adjusted for maximum visibility of M15 X-3.
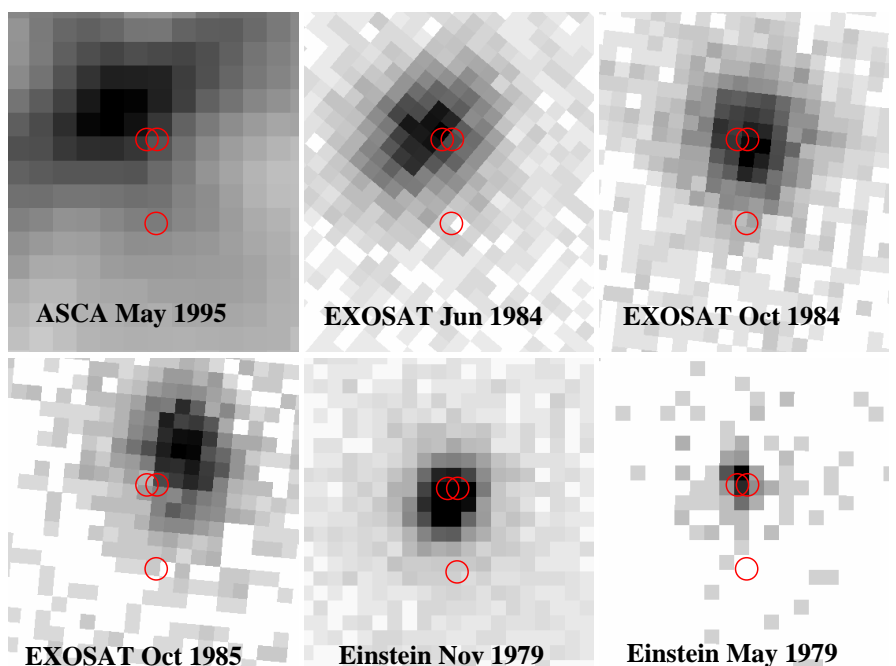

EXOSAT Jun 1984
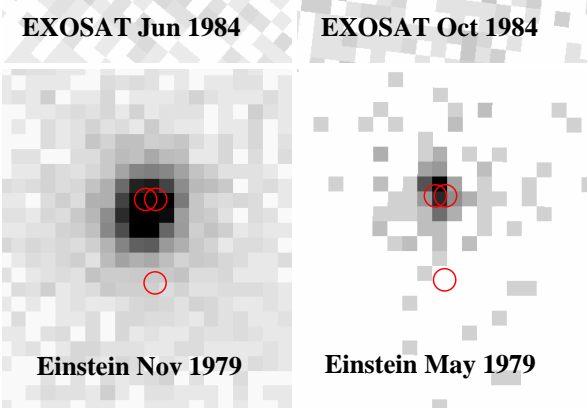

FIG. 7.- Postage stamp images of M15 from six imaging observations (ASCA, EXOSAT, and Einstein). The positions of both LMXBs and M15 X-3 are indicated with circles of radius 3". No boresight corrections have been applied to these images.

ple, conservative estimates of the upper limits in each case, we use a simple procedure. We compare the count rates in 5" circles around the peak of the observed source (taken to be AC211 and M15 X-2) and the expected location of M15 X-3 (after boresighting to align the detected source with the point midway between the bright LMXBs). We do not subtract the expected counts from the wings of the LMXBs' point spread function, as the shape of those PSFs are not necessarily well known; this makes our limits very conservative. We compute the 0.5 $10 \mathrm{keV} L_{X}$ for the M15 LMXB assuming a powerlaw of photon index 1.51, and multiply by the ratio of the counts in the two circles. The limits derived are given in Table 1 .

ASCA: One ASCA observation is available. The position of a bright ASCA SIS source can be centroided within 12" after appropriate corrections to the astrometry (Gotthelf et al. 2000). We find that the position of the M15 X-ray source, according to ASCA, is 21:29:58.88, $+12: 10: 12.97,12$ " NE of the positions of the known LMXBs, and thus consistent.

EXOSAT CMA: EXOSAT CMA images from three

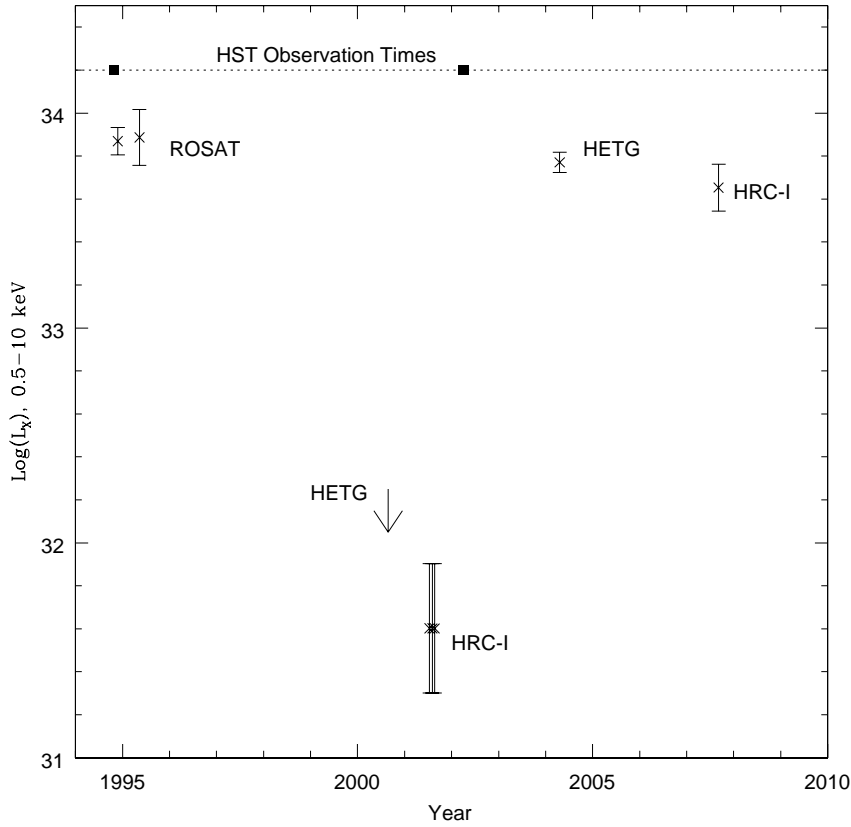

FIG. 8.- Timeline of sensitive X-ray detections and upper limits for M15 X-3, from the ROSAT HRI and Chandra HETG and HRCI imaging. The times of two useful HST observing epochs are also marked with squares.

epochs are available, each using the thin filter. The EXOSAT CMA provides positions accurate to within 8"12. The position of the single detected source is within 8" of AC211 and M15 X-2 in 2 of the observations, and 12" to the NW in the third observation.

Einstein HRI: Einstein HRI images for two epochs are available. Einstein positions are thought to be accurate to $3.2^{\prime \prime}$ (Grindlay et al. 1984), and the detected M15 source is consistent with the positions of either bright M15 LMXB.

\subsection{X-ray Conclusions}

The ROSAT and Chandra data require at least two substantial changes in flux; a decrease between 1995 and 2000, and an increase between 2001 and 2004 (Fig. 8). Consecutive X-ray observations tend to find the source in the same state, suggesting that typical on and off periods for M15 X-3 are a few years in duration. Further high-resolution monitoring observations would be greatly useful in constraining M15 X-3's recurrence history.

Our conclusion from the analysis of archival X-ray datasets on M15 is that if M15 X-3 has reached high $L_{X}$ s of $>10^{35} \mathrm{ergs} / \mathrm{s}$, it has done so rarely. The twelve most sensitive X-ray observations over 29 years all constrain M15 X-3 to $L_{X}<10^{35} \mathrm{ergs} / \mathrm{s}$. We consider three scenarios for M15 X-3's mass transfer rate (we assume a $10-\mathrm{km}, 1.4 M_{\odot} \mathrm{NS}$ accretor for each): a) the observations in hand are representative, and it spends $\sim$ half of its time "on" at $L_{X}=6 \times 10^{33} \mathrm{ergs} / \mathrm{s}$; for this case, $\dot{M} \sim 2 \times 10^{-13} M_{\odot} /$ year. b) M15 X-3 has undergone outbursts in the past 12 years, which we have happened to miss. The RXTE ASM coverage indicates no outbursts brighter than $\sim 3 \times 10^{36} \mathrm{ergs} / \mathrm{s}$ in the past 12

\footnotetext{
12 http://heasarc.gsfc.nasa.gov/docs/exosat/exosat_about.html
} 
years (apart from the 2007 brightening, 33.1 ), so we assume a maximum $L_{X}=3 \times 10^{36} \mathrm{ergs} / \mathrm{s}$. The twelve most sensitive existing X-ray datasets constrain M15 X-3 to $L_{X}<10^{35} \mathrm{ergs} / \mathrm{s}$; so we assume that X-3 is in outburst at $L_{X}<3 \times 10^{36} \mathrm{ergs} / \mathrm{s}$ only $1 / 12$ of the time. This gives a mass transfer rate of $\dot{M}<2 \times 10^{-11} M_{\odot} /$ year. c) M15 X-3 has undergone major outbursts, but they were more than 12 years ago; in this case, we cannot constrain its mass transfer rate.

\section{OTHER WAVELENGTHS}

\subsection{Optical photometry}

M15 has been the target of numerous HST optical campaigns. M15 X-3's location 20" from the center of M15 reduces crowding, but unfortunately places it outside the field of view of most HST high-resolution instruments during pointings at the core. (The $0.1^{\prime \prime}$ pixel size of the WFPC2 Wide Field chips does not adequately resolve this crowded area.) Three epochs of imaging cover M15 X-3 with the Planetary Camera (PC) chip, or with ACS/WFC. Unfortunately, during the ACS observation epoch (2006), the orientation of the spacecraft placed bleeding columns from a nearby bright giant onto M15 $\mathrm{X}-3$ 's position. Thus we remain with only two usable epochs, in only three bands: F336W (1994), and F555W and F439W (2002); see Fig. 9, and Table 2 .

Since the 2002 data were obtained with dithering, we constructed drizzled reconstructions of the F555W and F439W PC fields. In order to generate approximate color-magnitude diagrams (CMDs), we performed aperture photometry on the drizzled frames and on a combined frame of F336W exposures. We applied the standard calibration for the STMAG system to the resulting magnitudes. We then applied an aperture correction to an aperture of $0.5^{\prime \prime}$. We approximately aligned the resulting color-magnitude diagrams (CMDs) to the Johnson system by adding zeropoint shifts appropriate to a star at the main-sequence turnoff (MSTO); these shifts are neglible except for $\mathrm{F} 439 \mathrm{~W}$, which was increased by 0.60 mag. No color-dependent transformations were employed. The resulting CMDs are shown in Figs. 10 and 11. Given the use of aperture photometry without neighbor subtraction, we have not plotted the photometry for the region of radius $4^{\prime \prime}$ about the cluster center in order to produce cleaner fiducial sequences. Comparison with the $(V, B-V)$ CMD of van der Marel et al. (2002) shows reasonable alignment of the magnitude scales in the vicinity of the horizontal branch and main sequence turnoff.

We use the relative positions of M15 X-3 vs. AC211 given in Table 3 to derive a $0.14^{\prime \prime}(2 \sigma)$ error circle for M15 X-3's optical counterpart in the HST images. We have found a likely counterpart, within $0.05^{\prime \prime}(<1 \sigma)$ of the expected position, that shows evidence of possibly transient UV excess associated with phases of higher mass transfer. The 2002 data show a clear detection in $V$, but only a marginal detection in $B$ (Fig. 9), making the $B$ photometry of this object challenging. Since it was well below the automatic detection threshold in $B$, it had to be handled as a special case. The resulting $B-V$ color index puts the object well redward of the main sequence but with very large errors that include the main sequence (Fig. 10). As the 2002 HST data were

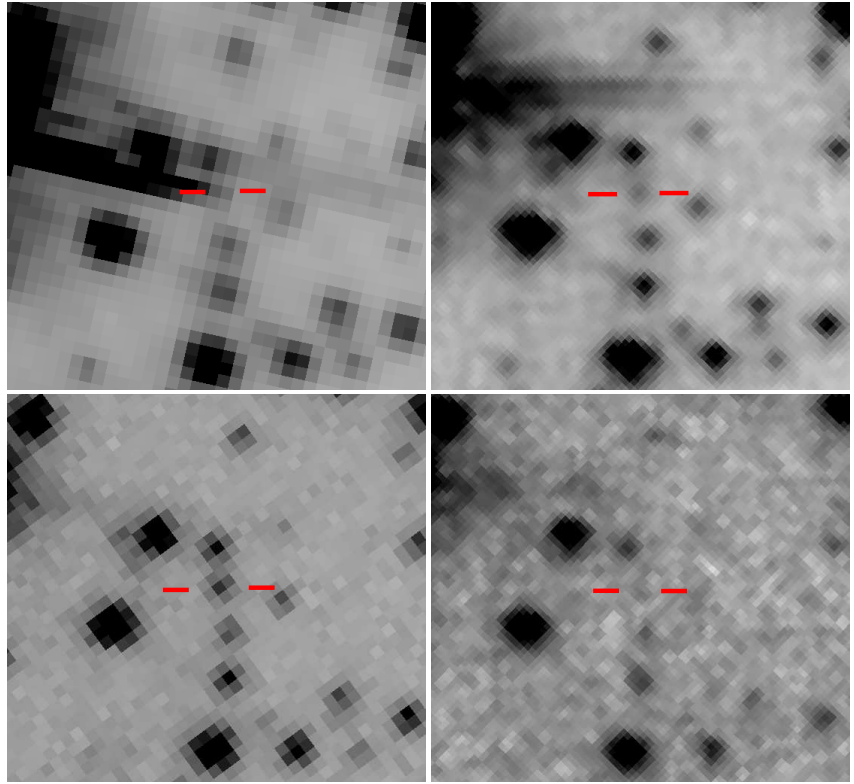

FIG. 9.- Finding charts for candidate counterpart of M15 X3. The location of M15 X-3 is bracketed by (red) dashes in each frame. Upper left, ACS/WFC F606W image from 2006 (affected by column bleeding); upper right, WFPC2 PC F555W image (2002); lower left, WFPC2 PC F336W image (1994); lower right, WFPC2 PC F439W image (2002)

taken only 8 months after the 2001 HRC observations that found M15 X-3 in quiescence, it seems likely that it was also in quiescence in 2002. In contrast, comparison of the $1994 \mathrm{U}$ and $2002 \mathrm{~V}$ data implies a clear UV excess in 1994 (see Fig. 11), relative to the main sequence, consistent with a mass transfer event. This makes sense, considering that the 1994 HST image was taken only one month before a ROSAT observation that found M15 X-3 to be active (although M15 X-3 could have varied during that month). 


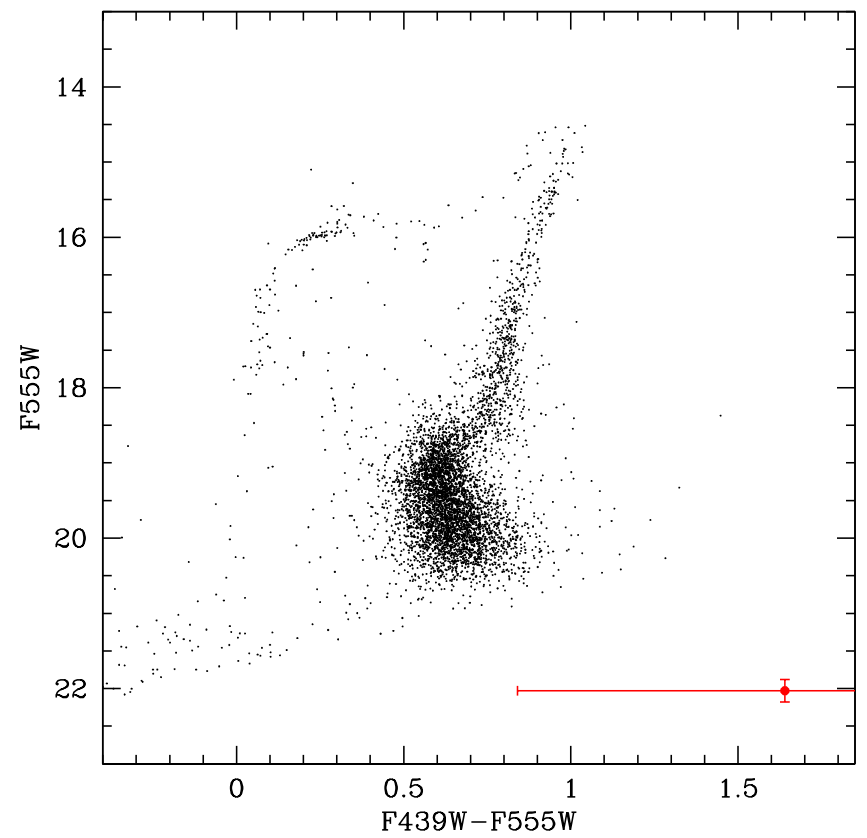

FIG. 10.- Color-magnitude diagram for M15, in F439W (" $B$ ") and F555W (" $V$ ") filters, both from the same observation epoch in 2002. (The calibration is described in the text and includes a zeropoint shift of $0.6 \mathrm{mag}$ in $\mathrm{F} 439 \mathrm{~W}$ to bring it closer to $B$.) The proposed counterpart to M15 X-3 is indicated by the red dot with the large error bars to the red of the main sequence (though consistent with it). 


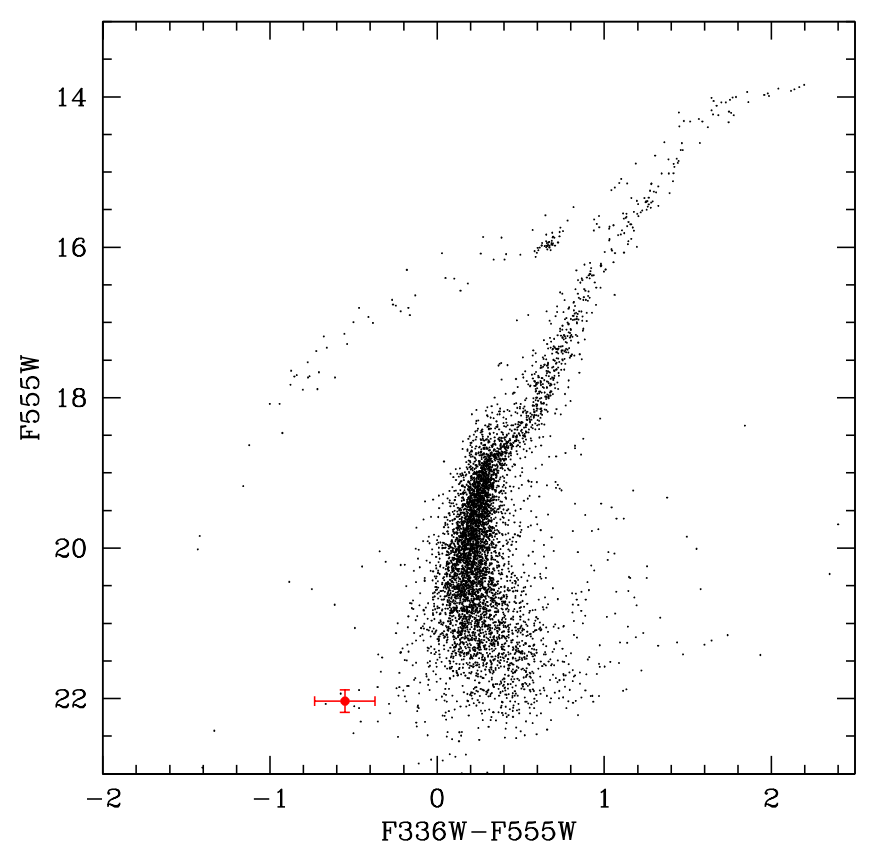

FIG. 11. - Color-magnitude diagram for M15, in F336W ("U") and F555W ("V") filters. (The calibration is described in the text.) The proposed counterpart to M15 X-3 is indicated by the red dot with error bars to the blue of the main sequence. Note that the F555W and F336W frames were taken in 2002 and 1994 respectively.

\subsection{Radio images}

The position of M15 X-3 lies in the field of view of four VLA observations reported in the literature. A 1.4 $\mathrm{GHz}$ image by Kulkarni et al. (1990) shows no emission from M15 X-3's location, for an upper limit of $0.3 \mathrm{mJy}$ $(4 \sigma)$ on October 11-12 1988. A deeper observation at $1.4 \mathrm{GHz}$ between April 9-14, 1990, by Johnston et al. (1991) allows an upper limit of $\sim 130 \mu \mathrm{Jy}(3 \sigma)$. An 8.4 GHz map from Feb. 11, 1991, by Knapp et al. (1996), finds a marginal $3 \sigma$ detection $(\sim 70 \mu \mathrm{Jy})$ at a position consistent with M15 X-3 (within 2"; the VLA was in hybrid $\mathrm{C} / \mathrm{D}$ configuration, with a larger beam). A fourth VLA observation on Oct. 13, 2004 at $8.6 \mathrm{GHz}$, reported by Bash et al. (2008), allows an upper limit of $25 \mu \mathrm{Jy}$ $(4 \sigma)$.

\section{DISCUSSION}

\subsection{Nature of the accretor}

M15 X-3's position near the center of M15 strongly indicates that it is a cluster member. M15 X-3's X-ray luminosity measurements fall within a range that is reached by both quiescent LMXBs and cataclysmic variables. We provide three arguments for why M15 X-3 is more likely to be an accreting neutron star:

Strong X-ray variability: M15 X-3's X-ray flux declined by two orders of magnitude between 1995 and 2000, and increased again to its prior level between 2001 and 2004. Such strong X-ray variability is a hallmark of LMXBs, but is rare among most cataclysmic variables. Baskill et al. (2005) observe that nonmagnetic CVs vary by one order of magnitude or less between observations, and do not reach maximum X-ray luminosities above $3 \times 10^{32} \mathrm{ergs} / \mathrm{s}$. The X-ray luminosities of polars do vary by two orders of magnitude on appropriate timescales, but they do not reach above $10^{33} \mathrm{ergs} / \mathrm{s}$ (Ramsav et al. 2004). Intermediate polars do attain $L_{X}>3 \times 10^{33}$ $\operatorname{ergs} / \mathrm{s}$, but they rarely show substantial long-term variability (Muno et al. 2004).

$X$-ray to optical flux ratio: The lack of simultaneity complicates this comparison. However, the 1994 ROSAT HRI observation which found M15 X-3 in its high state was only one month away from the HST WFPC2 F336W observations discussed in 4.1. Considering that M15 $\mathrm{X}-3$ seems to remain in each state for at least a year, we assume that these values can be compared. M15 X3's $V$ magnitude in 1994 must have been $>21$ (otherwise it would not be blue during outburst, given the $U$ constraint). Using $\log \left(F_{\text {Opt }}\right)=-0.4 \mathrm{~V}-3.96$, we find $F_{X}(0.5-2.5) / F_{O p t}=0.5$, which is higher than for most CVs (Verbunt et al. 1997). M15 X-3's relatively high Xray to optical flux ratio suggests a neutron star accretor.

Variation in $X$-rays vs. optical: To within a large uncertainty in the $B$ magnitude, M15 X-3 is consistent with being on or near the main sequence in the $(V, B-V)$ CMD, and thus is unlikely to be much fainter in quiescence. Our outburst $U$ detection thus limits the total $V$ increase in outburst to one magnitude, while the $\mathrm{X}$-ray flux varies by two orders of magnitude. Most CVs show larger variations in optical than X-ray (e.g. Garnavich \& Szkodv 1988).

These arguments suggest that M15 X-3 contains an accreting neutron star, although confirmation (through, e.g., X-ray spectroscopy in quiescence) is desirable. If M15 X-3 contains a neutron star, the observed outbursts are among the faintest seen from neutron star accretors, identifying M15 X-3's behavior as that of a very faint $\mathrm{X}$-ray transient.

\subsection{Very faint X-ray transients}

Very faint X-ray transients (VFXTs) are identified as X-ray transients with peak X-ray luminosities of $L_{X}=10^{33.5-34}$ to $10^{36} \mathrm{ergs} / \mathrm{s}$ and quiescent luminosities at least 10 times fainter (Muno et al. 2005b; Sakano et al. 2005; Wiinands et al. 2006). Thirty systems showing such behavior have been identified, with 3-4 of them being active at any moment in the Galactic Center. Some rise up to $L_{X} \sim 10^{35} \mathrm{ergs} / \mathrm{s}$, with a low $(\lesssim 10 \%)$ duty cycle, while others, such as XMMU J174716.1-281048 and CXOGC J174535.5-290124, maintain $L_{X} \sim 10^{34} \mathrm{ergs} / \mathrm{s}$ with relatively high $(\sim 50 \%)$ duty cycles (Muno et al. 2005b; Del Santo et al. 2007; Degenaar \& Wijnands 2008). M15 X-3 appears to show similar behavior to the latter group, being observed to be "on" at $L_{X} \sim 6 \times 10^{33} \mathrm{ergs} / \mathrm{s}$ in half of the X-ray observations with sufficient sensitivity to detect such a state.

Typical, better-understood X-ray transients have peak X-ray luminosities of $L_{X}=10^{36}$ to $10^{38} \mathrm{ergs} / \mathrm{s}$, and timeaveraged mass transfer rates of $\dot{\mathrm{M}} \gtrsim 10^{-11} M_{\odot} /$ year. The VFXTs, by contrast, show combinations of duty cycles and peak $L_{X}$ s which indicate time-averaged mass transfer rates one to two orders of magnitude lower than $10^{-11}$ $M_{\odot} /$ year, difficult to understand in a binary evolution context (King \& Wiinands 2006). We note that VFXTs may undergo normal outbursts at other (so far unobserved) times, and thus might not have particularly low 
time-averaged mass transfer rates. In either case, their unusually low observed accretion luminosities are difficult to understand within the standard disk instability model, and thus warrant study.

Several possible explanations for VFXT behavior can be immediately ruled out for M15 X-3 (though they may be relevant for other VFXTs). Accretion from the wind of a massive (e.g. B) star or a subgiant (Pfahl et al. 2002) can be ruled out for an optically faint star in a globular cluster. An intermediate-mass black hole accretor (King \& Wijnands 2006) can also be ruled out, due to M15 X-3's offset from M15's core, and the expected rapid settling of massive objects into the core.

If our observations are representative, we can infer a time-averaged mass transfer rate of $\dot{M} \sim 2 \times 10^{-13}$ $M_{\odot} /$ year (3.4). This is roughly the minimum masstransfer rate achievable through binary evolution of a main-sequence star with a neutron star companion after a Hubble time (King \& Wijnands 2006), suggesting an extremely low-mass brown dwarf companion might be a possible solution. However, our HST photometry suggests (with large uncertainties) that the companion to M15 X-3 is a main-sequence star with a mass of $\sim 0.65 M_{\odot}$ (using an isochrone from Briley et al. 2004). Confirmation of a main-sequence companion (through deeper high-resolution HST imaging) would clearly rule out an extremely low-mass substellar companion. It would also severely constrain possible models of accretion from the wind of a low-mass main sequence star, as Willems \& Kolb (2003) found a maximum time-averaged bolometric accretion luminosity of $10^{33} \mathrm{ergs} / \mathrm{s}$ from stars in this mass range in the pre-LMXB stage.

One plausible alternative for M15 X-3 is an extremely unfavorable inclination angle, such as the X-ray transient identified by Muno et al. (2005a), allowing an isotropic $\mathrm{X}$-ray luminosity higher by two orders of magnitude. At $10 \mathrm{kpc}$, an outburst reaching $10^{36} \mathrm{ergs} / \mathrm{s}$ might be predicted to reach radio fluxes of $20 \mu \mathrm{Jy}$ from a neutron star LMXB, or $1.0 \mathrm{mJy}$ from a black hole LMXB in the hard state (using the correlation from Migliari \& Fender 2006). The stringent Oct. 2004 radio upper limit of 25 $\mu \mathrm{Jy}, 6$ months after an X-ray observation showing activity, suggests that if M15 X-3 is highly inclined, it is more likely a neutron star LMXB. The possible $70 \mu \mathrm{Jy}$ radio detection in 1991, if real, suggests either a major 1991 outburst (unfortunately not covered with sensitive imaging), or that a large fraction of M15 X-3's accretion power at low accretion rates is going into the formation of jets (as found by Gallo et al. 2006, for black hole systems). A high-inclination explanation for the low $L_{X}$ might predict detectable eclipses (the 2004 lightcurve (Fig 5) excludes eclipses longer than $1000 \mathrm{~s}$ ), and that the neutron star should not be directly visible in quiescence as a blackbody-like X-ray source (testable with ACIS spectroscopy in quiescence).

A second alternative is the removal of most mass transferred from the companion out of the system, perhaps by the pressure of a fast-spinning NS magnetic field, the "propeller effect" (Illarionov \& Sunyaev 1975). Detailed simulations of the interaction of an accretion disk with such a spinning magnetic field find that some material does leak down onto the neutron star poles (Romanova et al. 2005). A moderately effective pro- peller effect may explain the particularly low mass transfer rates inferred for VFXTs. In this case, the dividing line between VFXTs and "normal" LMXBs may be ambiguous, dependent on spin rates, magnetic field strength and/or geometry. VFXTs may slowly accumulate sufficiently massive disks to produce normal outbursts. Some objects do show VFXT-like outbursts as well as brighter outbursts, e.g. GRS 1741.9-2853 and XMM J174457-2850.3 (Muno et al. 2003; Sakano et al. 2005; Degenaar \& Wijnands 2008), supporting this possibility.

A third alternative is variability in the mass transfer rates from the companion, on timescales greater than tens of years, which is thought to occur in cataclysmic variables (e.g. Hameury et al. 1989). (King \& Wiinands 2006) dismiss mass transfer cycles as an explanation for VFXTs as a class for two reasons: a lack of systems much brighter than the mean mass transfer rate to balance those which are fainter, and a lack of a mechanism that can deliver such strong variability. However, the known bright neutron star LMXBs tend to be brighter than evolutionary calculations predict at their orbital periods (Podsiadlowski et al. 2002; Pfahl et al. 2003). One very plausible driver of such variability is an irradiationdriven instability, wherein the X-ray irradiation of the secondary causes slow expansion, leading to mass transfer cycles on $\sim 10^{8}$ year timescales (Podsiadlowski 1991; Hameury et al. 1993; Ritter et al. 2000; Büning \& Ritter 2004).

\section{CONCLUSIONS}

We have identified a transient X-ray source in the globular cluster M15, denoted M15 X-3. This source has been detected at $L_{X}=4-8 \times 10^{33} \mathrm{ergs} / \mathrm{s}$ in 4 ROSAT and Chandra observations, and $L_{X} \sim 2-6 \times 10^{31} \mathrm{ergs} / \mathrm{s}$ in 3 Chandra HRC-I observations, with an apparently softer spectrum. Archival HST observations reveal a star with an apparent ultraviolet excess at the X-ray position. Within broad uncertainty, due to a very weak detection in $B$, the location of the proposed counterpart in the $(V$, $B-V)$ CMD is consistent with it being a main-sequence star. The X-ray and optical characteristics of this source suggest an accreting neutron star nature, with the Xray flux history resembling those of "very faint X-ray transients" seen in the Galactic Center region. A 1991 VLA observation may have detected M15 X-3 at $\sim 70$ $\mu \mathrm{Jy}$. If M15 X-3 consists of a neutron star accreting from a main-sequence star, as the evidence suggests, the observed accretion rate onto the NS must be rather lower than the expected mass-transfer rate for a Roche-lobe filling system.

The location of this object is a mixed blessing. Being located in M15, 20" from two luminous and persistent X-ray binaries, hides this object from all X-ray instruments but those with the highest angular resolution (only Chandra for the foreseeable future). Being in such a dense globular cluster also prevents optical followup with any instrument with inferior angular resolution to HST. However, M15's intrinsic interest has provided us with numerous archival observations that allowed the discovery of M15 X-3. Most importantly, the low extinction in this direction allows future study of this object in quiescence, which is excluded for all other known very faint X-ray transients both in the optical/UV and soft X-rays. 
M15 X-3 may be a key object for understanding the nature of very faint $\mathrm{X}$-ray transients.

We thank H. Tananbaum and the CXC for granting the DDT observation ObsID 9584 of M15. COH warmly thanks M. Muno, R. Wijnands, J. Miller-Jones and T. J. Maccarone for discussions, and V. Kashyap for HRC-I RMF calculations. COH acknowledges the Lindheimer Postdoctoral Fellowship at Northwestern University, Chandra grant G07-8078X to the University of Virginia, and funding from the University of Alberta Physics
Department. This research has made use of data obtained through the HEASARC online service, provided by the NASA/Goddard Space Flight Center. RXTE AllSky Monitor results are provided by the ASM/RXTE team. This work is based in part on observations made with the NASA/ESA Hubble Space Telescope, obtained from the data archive at the Space Telescope Science Institute. STScI is operated by the Association of Universities for Research in Astronomy, Inc., under NASA contract NAS 5-26555.

\section{REFERENCES}

Anderson, S. B. 1993, PhD thesis, AA(California Inst. of Tech., Pasadena.)

Bash, F. N., Gebhardt, K., Goss, W. M., \& Vanden Bout, P. A. 2008, AJ, 135, 182

Baskill, D. S., Wheatley, P. J., \& Osborne, J. P. 2005, MNRAS, 357,626

Baumgardt, H., Hut, P., Makino, J., McMillan, S., \& Portegies Zwart, S. 2003, ApJ, 582, L21

Briley, M. M., Harbeck, D., Smith, G. H., \& Grebel, E. K. 2004, AJ, 127,1588

Brown, E. F., Bildsten, L., \& Rutledge, R. E. 1998, ApJ, 504, L95

Büning, A., \& Ritter, H. 2004, A\&A, 423, 281

Cameron, P. B., Rutledge, R. E., Camilo, F., Bildsten, L., Ransom, S. M., \& Kulkarni, S. R. 2007, ApJ, 660, 587

Campana, S., Colpi, M., Mereghetti, S., Stella, L., \& Tavani, M. 1998, A\&A Rev., 8, 279

Charles, P. A., Clarkson, W. I., \& van Zyl, L. 2002, New Astronomy, 7, 21

Cocchi, M., et al. 2001, A\&A, 378, L37

Cornelisse, R., Verbunt, F., in't Zand, J. J. M., Kuulkers, E., \& Heise, J. 2002, A\&A, 392, 931

Davis, J. E. 2001, ApJ, 562, 575

Degenaar, N., \& Wijnands, R. 2008, ArXiv e-prints, 807

Del Santo, M., Sidoli, L., Mereghetti, S., Bazzano, A., Tarana, A., \& Ubertini, P. 2007, A\&A, 468, L17

Deutsch, E. W., Margon, B., \& Anderson, S. F. 1998, AJ, 116, 1301

Dieball, A., Knigge, C., Zurek, D. R., Shara, M. M., Long, K. S., Charles, P. A., \& Hannikainen, D. 2007, ApJ, 670, 379

Dull, J. D., et al. 1997, ApJ, 481, 267

-. 2003, ApJ, 585, 598

Gallo, E., et al. 2006, MNRAS, 370, 1351

Garnavich, P., \& Szkody, P. 1988, PASP, 100, 1522

Gerssen, J., van der Marel, R. P., Gebhardt, K., Guhathakurta, P., Peterson, R. C., \& Pryor, C. 2002, AJ, 124, 3270

Gotthelf, E. V., Ueda, Y., Fujimoto, R., Kii, T., \& Yamaoka, K. 2000, ApJ, 543, 417

Grindlay, J. E., Heinke, C., Edmonds, P. D., \& Murray, S. S. 2001, Science, 292, 2290

Grindlay, J. E., Hertz, P., Steiner, J. E., Murray, S. S., \& Lightman, A. P. 1984, ApJ, 282, L13

Guhathakurta, P., Yanny, B., Schneider, D. P., \& Bahcall, J. N. 1996, AJ, 111, 267

Haensel, P., \& Zdunik, J. L. 1990, A\&A, 227, 431

Hameury, J. M., King, A. R., \& Lasota, J. P. 1989, MNRAS, 237, 39

Hameury, J. M., King, A. R., Lasota, J. P., \& Raison, F. 1993, A\&A, 277, 81

Hands, A. D. P., Warwick, R. S., Watson, M. G., \& Helfand, D. J. 2004, MNRAS, 351, 31

Hannikainen, D. C., et al. 2005, MNRAS, 357, 325

Heinke, C. O., Cohn, H. N., \& Lugger, P. M. 2007, The Astronomer's Telegram, 1211, 1
Heinke, C. O., Edmonds, P. D., \& Grindlay, J. E. 2001, ApJ, 562,363

Heinke, C. O., Rybicki, G. B., Narayan, R., \& Grindlay, J. E. 2006, ApJ, 644, 1090

Hertz, P., \& Grindlay, J. E. 1983, ApJ, 275, 105

Illarionov, A. F., \& Sunyaev, R. A. 1975, A\&A, 39, 185

Janulis, R. 1992, Baltic Astronomy, 1, 25

Johnston, H. M., Kulkarni, S. R., \& Goss, W. M. 1991, ApJ, 382, L89

Jonker, P. G., Wijnands, R., \& van der Klis, M. 2004, MNRAS, 349,94

King, A. R. 2000, MNRAS, 315, L33

King, A. R., \& Wijnands, R. 2006, MNRAS, 366, L31

Knapp, G. R., Gunn, J. E., Bowers, P. F., \& Vasquez Poritz, J. F. 1996, ApJ, 462, 231

Kulkarni, S. R., Narayan, R., \& Romani, R. W. 1990, ApJ, 356, 174

Lauer, T. R., et al. 1991, ApJ, 369, L45

Liedahl, D. A., Osterheld, A. L., \& Goldstein, W. H. 1995, ApJ, 438, L115

Migliari, S., \& Fender, R. P. 2006, MNRAS, 366, 79

Muno, M. P., et al. 2004, ApJ, 613, 1179

Muno, M. P., Baganoff, F. K., \& Arabadjis, J. S. 2003, ApJ, 598, 474

Muno, M. P., et al. 2005a, ApJ, 633, 228

Muno, M. P., et al. 2005b, ApJ, 622, L113

Pfahl, E., Rappaport, S., \& Podsiadlowski, P. 2002, ApJ, 571, L37

—. 2003, ApJ, 597, 1036

Podsiadlowski, P. 1991, Nature, 350, 136

Podsiadlowski, P., Rappaport, S., \& Pfahl, E. D. 2002, ApJ, 565, 1107

Ramsay, G., Cropper, M., Wu, K., Mason, K. O., Córdova, F. A., \& Priedhorsky, W. 2004, MNRAS, 350, 1373

Ritter, H., Zhang, Z.-Y., \& Kolb, U. 2000, A\&A, 360, 969

Romanova, M. M., Ustyugova, G. V., Koldoba, A. V., \&

Lovelace, R. V. E. 2005, ApJ, 635, L165

Sakano, M., Warwick, R. S., Decourchelle, A., \& Wang, Q. D. 2005, MNRAS, 357, 1211

Sosin, C., \& King, I. R. 1997, AJ, 113, 1328

van den Bosch, R., de Zeeuw, T., Gebhardt, K., Noyola, E., \& van de Ven, G. 2006, ApJ, 641, 852

van der Marel, R. P., Gerssen, J., Guhathakurta, P., Peterson, R. C., \& Gebhardt, K. 2002, AJ, 124, 3255

Verbunt, F., Bunk, W. H., Ritter, H., \& Pfeffermann, E. 1997, A\&A, 327, 602

Verbunt, F., Elson, R., \& van Paradijs, J. 1984, MNRAS, 210, 899

White, N. E., \& Angelini, L. 2001, ApJ, 561, L101

Wijnands, R., et al. 2006, A\&A, 449, 1117

Wijnands, R., Rol, E., Cackett, E. M., Starling, R. L. C., \&

Remillard, R. A. 2007, astro-ph/0709.0061, 709

Willems, B., \& Kolb, U. 2003, MNRAS, 343, 949 
TABLE 1

Summary of X-ray Observations of M15 X-3

\begin{tabular}{lcccc}
\hline \hline \multicolumn{1}{c}{ OBS_ID } & Obs. Date & Exposure & Instrument & $L_{X}$ \\
\hline $4879-4882$ & 1979 Nov 19 & 5.07 & Einstein-HRI & $<3.0 \mathrm{e} 34$ \\
656 & 1978 Nov 22 & 0.26 & Einstein-HRI & $<1 \mathrm{e} 35$ \\
657 & 1979 May 17 & 1.60 & Einstein-HRI & $<3 \mathrm{e} 34$ \\
\hline 28831 & 1984 Jun 30 & 23.73 & EXOSAT-CMA & $<3 \mathrm{e} 35$ \\
37046 & 1984 Oct 22 & 36.15 & EXOSAT-CMA & $<9 \mathrm{e} 34$ \\
63182 & 1985 Oct 20 & 20.27 & EXOSAT-CMA & $<1.6 \mathrm{e} 35$ \\
\hline 400081 N00 & 1992 May 16 & 8.79 & ROSAT-PSPC & $<3.4 \mathrm{e} 34$ \\
400611 N00 & 1994 Nov 27 & 52.47 & ROSAT-HRI & $7.4 \pm 1 \times 10^{33}$ \\
400611 A01 & 1995 May 11 & 27.62 & ROSAT-HRI & $7.7 \pm 2 \mathrm{e} 33$ \\
\hline 43042000 & 1995 May 16 & 37.38 & ASCA-SIS & $<2.7 \mathrm{e} 36$ \\
\hline 675 & 2000 Aug 24 & 20.03 & CXO-HETGS & $<1.8 \mathrm{e} 32$ \\
1903 & 2001 Jul 13 & 9.10 & CXO-HRC-I & $2 \times 10^{31}$ \\
2412 & 2001 Aug 03 & 8.90 & CXO-HRC-I & $2 \times 10^{31}$ \\
2413 & 2001 Aug 22 & 10.87 & CXO-HRC-I & $2 \times 10^{31}$ \\
4572 & 2004 Apr 17 & 60.14 & CXO-HETGS & $5.9 \times 10^{33}$ \\
9584 & 2007 Sep 05 & 2.14 & CXO-HRC-I & $4.5 \times 10^{33}$
\end{tabular}

Note. - Times in kiloseconds. Unabsorbed $L_{X}(0.5-10 \mathrm{keV})$ observed, or upper limits reached (see text), in each observation. We use PIMMS and a powerlaw of photon index 1.5 absorbed by $N_{H}=4.6 \times 10^{20} \mathrm{~cm}^{-2}$ to estimate detections or upper limits for observations where $L_{X}$ may be $>10^{33} \mathrm{ergs} / \mathrm{s}$, or PIMMS and a blackbody of $0.135 \mathrm{keV}$ with the same $N_{H}$ for observations where $L_{X}$ is constrained to $<10^{33} \mathrm{ergs} / \mathrm{s}$.

TABLE 2

Summary of HST Observations

\begin{tabular}{lcccc}
\hline \hline Prog. ID & Epoch & Instrument & Exposures & Magnitude \\
\hline 5742 & 1994 Oct 26 & WFPC2 & $3 \times 600$ F336W & $21.5 \pm 0.2$ \\
9039 & 2002 Apr 5 & WFPC2 & $12 \times 16$ F555W & $22.0 \pm 0.2$ \\
9039 & 2002 Apr 5 & WFPC2 & $4 \times 40$ F439W & $23.7 \pm 0.8$
\end{tabular}

Note. - Magnitudes refer to proposed M15 X-3 counterpart, with calibration as discussed in text.

TABLE 3

Chandra Source List

\begin{tabular}{lccccrr}
\hline \hline \multicolumn{1}{c}{ Name } & RA & Dec & Cts: 2000 & Cts: 2001 & Cts: 2004 & Cts: 2007 \\
\hline AC211 & $21: 29: 58.310$ & $12: 10: 02.85$ & - & $80769(299)$ & - & $4735(70)$ \\
M15 X-2 & $21: 29: 58.124(1)$ & $12: 10: 02.37(1)$ & - & $191255(454)$ & - & $13960(120)$ \\
DN, src C & $21: 29: 57.323(6)$ & $12: 10: 43.9(1)$ & {$[5]$} & $21(5)$ & $79(10)$ & $<1$ \\
PN, K648 & $21: 29: 59.40(1)$ & $12: 10: 27.6(1)$ & $<2$ & $9(3)$ & {$[5]$} & $<1$ \\
M15 X-3 & $21: 29: 58.155(3)$ & $12: 09: 40.04(6)$ & $<2$ & $8(3)$ & $480(23)$ & $31(6)$
\end{tabular}

NoTE. - Positions and counts from WAVDETECT, in 0.5-7 keV band for ACIS data, full band for HRC data. Nondetections indicated with upper limits, subjective detections in brackets. Errors are in parentheses, on the last quoted digit, and include only random (not possible systematic) errors. The exposure times for the observations are: 2000 ACIS-S/HETGS, $20.03 \mathrm{ks} ; 2001$ HRC-I, 28.87 ks; 2004 ACIS-S/HETGS, 60.14 ks; 2007 HRC-I, 2.14 ks.

TABLE 4

Spectral Fits to M15 X-3, 2004 CXO

\begin{tabular}{cccccc}
\hline \hline Model & $N_{H} \times 10^{20}$ & $\Gamma$ & $\chi_{\nu}^{2} /$ dof & $L_{X}$ & $L_{X, N S}$ \\
\hline Pow & $4.6^{*}$ & $1.49 \pm 0.13$ & $0.89 / 29$ & $5.9_{-0.6}^{+0.4} \times 10^{33}$ & - \\
Pow+NSATMOS & $4.6^{*}$ & $1.42_{-0.25}^{+0.18}$ & $0.90 / 28$ & $5.8_{-0.6}^{+0.5} \times 10^{33}$ & $1.7_{-1.7}^{+4.7} \times 10^{32}$ \\
MEKAL & $4.6^{*}$ & - & $0.96 / 29$ & $5.9_{-0.5}^{+0.7} \times 10^{33}$ & $82_{-82}^{+26}$ \\
& & & & & $14_{-5}^{+17}$
\end{tabular}

NoтE. - Power-law, power-law plus neutron star atmosphere, and mekal model fits to spectrum of M15 X-3 in 2004. Errors are $90 \%$ confidence for a single parameter. X-ray luminosities for $0.5-10 \mathrm{keV}$, in ergs/s. $k T$ in eV for NSATMOS model, keV for MEKAL model.

*: fixed to the assumed cluster value. 